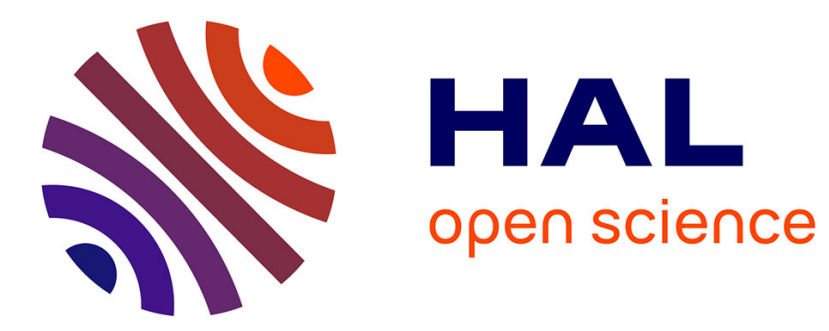

\title{
Dispersion curve measurement using Talbot bands
}

\author{
M. Warenghem, C.P. Grover
}

\section{To cite this version:}

M. Warenghem, C.P. Grover. Dispersion curve measurement using Talbot bands. Revue de Physique Appliquée, 1988, 23 (6), pp.1169-1178. 10.1051/rphysap:019880023060116900 . jpa-00245922

\section{HAL Id: jpa-00245922 https://hal.science/jpa-00245922}

Submitted on 1 Jan 1988

HAL is a multi-disciplinary open access archive for the deposit and dissemination of scientific research documents, whether they are published or not. The documents may come from teaching and research institutions in France or abroad, or from public or private research centers.
L'archive ouverte pluridisciplinaire HAL, est destinée au dépôt et à la diffusion de documents scientifiques de niveau recherche, publiés ou non, émanant des établissements d'enseignement et de recherche français ou étrangers, des laboratoires publics ou privés. 
Classification

Physics Abstracts

78.20D $-78.20 \mathrm{~F}-61.30$

\title{
Dispersion curve measurement using Talbot bands
}

\author{
M. Warenghem and C. P. Grover (1) \\ Laboratoire de Dynamique des Cristaux Moléculaires, U.S.T.L.F.A., U.F.R. de Physique, 59655 Villeneuve \\ d'Ascq Cedex, France \\ (1) Physics Division, National Research Council, Montreal Rd, Ottawa K1A OR6, Canada
}

(Reçu le 9 novembre 1987, révisé le 16 février 1988, accepté le 8 mars 1988)

\begin{abstract}
Résumé. - Les bandes de Talbot, ressemblant à un spectre cannelé, sont observables lorsqu'une lame de phase est partiellement insérée dans le champ optique d'un spectroscope. Leur position dans le spectre de la source est directement liée au retard optique introduit par la lame de phase et donc à son indice de réfraction. Dans le cas d'un spectroscope à réseau, les bandes n'existent que dans les ordres de diffraction situés du côté par lequel la lame a été insérée. Les bandes apparaissent également lorsque la lame de phase est constituée de deux parties d'indices différents, du côté de la lame d'indice le plus élevé. En utilisant des technologies modernes de mesures sur ce phénomène connu de longue date, nous proposons une nouvelle méthode pour obtenir la courbe de dispersion de matériaux transparents. Elle est basée sur une détermination précise de la position des bandes dans le spectre associée à une manipulation numérique des données conduisant à l'obtention de la courbe de dispersion et ce, en une seule expérience alors que la technique classique en nécessite autant que de points sur la courbe. Dans cette publication, après avoir décrit la méthode, nous reportons des résultats expérimentaux obtenus sur des substances tests, démontrant ainsi la faisabilité et la fiabilité de la méthode. Ensuite elle est étendue aux matériaux biréfringents. Il est montré qu'il est possible de mesurer les courbes de dispersions ordinaires et extraordinaires simultanément. Des résultats expérimentaux concernant les cristaux liquides sont également reportés.
\end{abstract}

\begin{abstract}
Talbot bands, looking like a channelled spectrum, are produced when a phase plate is partly inserted in the field of a spectroscope. The position and the spacing of the bands in the spectrum of the source directly depends on the optical thickness and therefore the refractive index of the phase plate. In the case of a transmission grating spectroscope, the bands appear only in spectral orders on the same side as the phase plate. The bands can be observed also in case of a two stepped plate, their position being related to the difference of the two refractive indices of the plate, they appear in spectrum orders on the side where the higher index half plate have been inserted in. Using this early known phenomenon in conjunction with modern technology, we propose a method based upon an accurate determination of the positions of these dark bands for computing the dispersion curve of any transparent liquid or solid material, the curve being built up from only one experiment whereas several are required usually. In this paper, after the method have been described, experimental results obtained with known glasses are reported proving the efficiency and the reliability of the technique. Also this method is extended to birefringent materials, it is shown that the extraordinary and ordinary dispersion curve can be obtained simultaneously. Experimental results on liquid crystals materials are reported.
\end{abstract}

Apart from few original methods using for example Fourier Holography [1], there exist, generally speaking, two ways for measuring the wavelength dependence of the refractive index the dispersion curve - for a material. This curve can be obtained from either several experiments using monochromatic sources or one experiment using a white light source. A very high accuracy can be obtained with the former technique so long as experimental parameters such as the temperature and the molecular orientation in the case of liquid crystals can be easily reproduced from an experiment to another one. The second method involves the channeled spectrum technique and thus it necessitates a more sophisticated experimental set up combining both an interferometric apparatus and a spectroscope. In this paper we propose a method halfway between the two previous one, it consists in 
using some modern technology in conjunction with a phenomenon observed as early as early as 1837 but rather neglected so far.

As first observed by Talbot [2], some dark bands appear in the spectrum of a spectroscope illuminated with a white light source when a phase plate is partially inserted in the field of the spectroscope. The position and the spacing of the bands in the spectrum directly depends on the index of refraction of the plate. On the other hand, the calibration of the spectroscope allowes the abscissa of a band to be expressed in terms of wavelength. Therefore every dark band is associated with both an index value and the corresponding wavelength, in other words, every dark band is associated with a data point on the dispersion curve of the material the phase plate is made of.

The presented method resulting in this dispersion curve determination for a given material consists of three steps. After the spectroscope has been calibrated in the first step, the plate to be studied is inserted close to the grating and upstream with respect to it. Half the field covered with the plate insures the best visibility of the bands. Then, the curve of the output intensity versus the angular abscissa is stored by means of a photosensor mounted on a high precision translation stage. In the last step, these data are processed for the dark bands position to be determined as well as the corresponding wavelength and there from the associated index of refraction of the plate.

In the first section of this paper the relationship between the position of the bands and the index of refraction is derived from the theory of the bands as explained by Stokes [3] and Airy [4] and adapted to our apparatus (a Ronchi Ruling spectroscope ( $\left.{ }^{1}\right)$ ). The second part is devoted to this set up as well as the experimental results for a test material showing the reliability and the accuracy of the presented

$\left({ }^{1}\right)$ Every even diffraction order vanishes. method. Any isotropic transparent solid or liquid material can be studied using this technique. The case of the birefringent materials will be considered in the third paragraph. It will be shown that suitably choosing the polarisation of the input beam with regard to the optical axis direction of the sample, the two dispersion curves corresponding respectively to the ordinary and extraordinary index can be obtained either separately or simultaneously. Also experimental results on liquid crystals materials are presented in this section.

\section{Theory.}

The basic set up for observing the Talbot bands consists of a phase plate partially inserted in the field of a spectroscope illuminated with a white light source. As it has been found in the previous experiments $[2,8]$ either a prism or a grating can be used as the dispersion element of the spectroscope and the maximum visibility of the bands occurs while the phase plate is covering half the field of the grating or the prism. Subsequently, the case of a two stepped plate, each part covering half the field of a grating spectroscope will be considered, the existence and the visibility of the bands discussed as a function of the other parameters of the set up such as the phase plate size and the dispersion power of the grating.

For a monochromatic beam illuminating the spectroscope, the intensity observed in the screen (Fig. 1) can be determined by using the Fourier Transform technique. For a two stepped plate, each part covering $N$ lines of the grating and half the beam being delayed for $\phi$ with respect to the other, the intensity becomes :

$$
\begin{array}{r}
I=B_{0}\left(\sigma_{0}\right)\left(\frac{\sin 2 \pi u b}{2 \pi u b}\right)^{2}\left(\frac{\sin N u \pi p}{\sin \pi u p}\right)^{2} \times \\
\times[1+\cos (2 N \pi u p+\phi)]
\end{array}
$$

where $p$ is the period of the grating, $2 b$ its line width

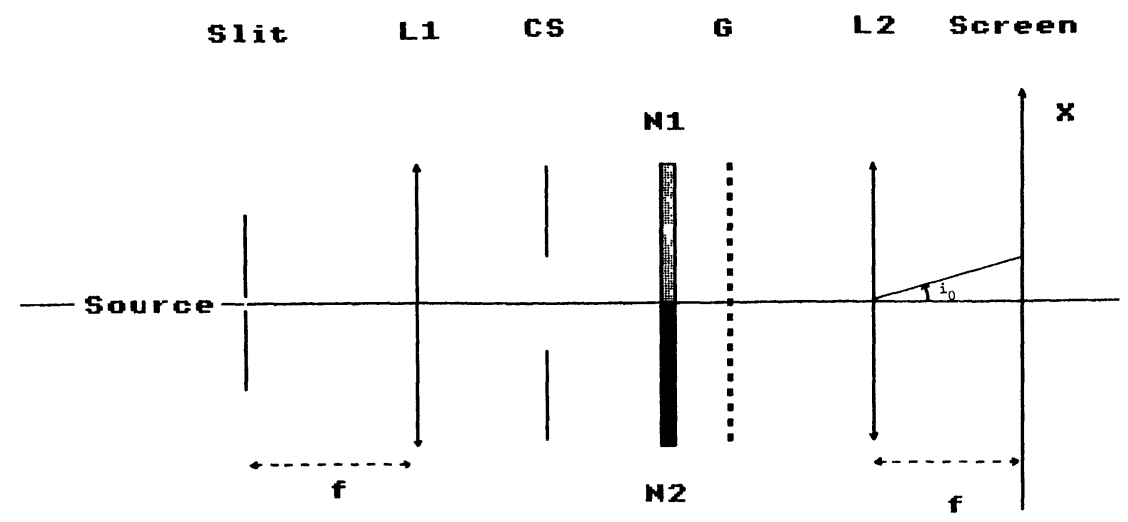

Fig. 1. - Basic set up for observing Talbot bands. $L_{1}, L_{2}$ : lenses ; C.S. : collimating slit; $N_{1}, N_{2}:$ sample index of refraction. $G$ : grating; $i_{0}$ : angular abscissa. 
and $u$ the spatial frequency defined as usual. In the case of a polychromatic input beam, this intensity has to be summed over the spectral range $\Delta \sigma$ of the source. This integral has been performed according to the Stokes and Airy method [3,4] consisting of integrating with respect to the spatial frequency change $\varepsilon$ due to the wavelength change for a given point in the screen of angular abscissa $i_{0}$, after the phase difference $\phi$ has been expanded as a function of the same variable $\varepsilon$. Mathematically this variable is defined as follows :

$$
\left\{\begin{array}{c}
\pi u p=\pi u_{0} p+\varepsilon \\
u_{0}=\sigma_{0} \sin i_{0}
\end{array}\right.
$$

where $i_{0}$ is the angular abscissa of the considered point on the screen. The wave number $\sigma_{0}$ is an arbitrary origin and it can always be chosen in such a manner that it would correspond to a maximum of intensity of the order $k$ of the grating at the considered point, then :

$$
u_{0}=\frac{k}{p} ; \quad \pi u p=k \pi+\varepsilon
$$

The phase difference has to be expanded with respect to the variable $\varepsilon$; let $A$ and $2 \mathrm{~B}$ be the first coefficients in this expansion :

$\phi=A+2 B \varepsilon+\cdots=\phi_{\left(u_{0}\right)}+\left(\frac{\delta \phi}{\mathrm{d} u}\right)_{u_{0}} \frac{\varepsilon}{\pi p} \cdots$

In case of a parallel plate, these coefficients can be expressed in terms of the refractive index $n$ of the plate, its thickness $d$ and the wavenumber $\sigma$ :

$$
\begin{aligned}
& \phi=2 \pi \sigma\left(n_{1}-n_{2}\right) d \\
& A=2 \pi \sigma_{0}\left(n_{1\left(\sigma_{0}\right)}-n_{2\left(\sigma_{0}\right)}\right) d \\
& B=\frac{A}{2 k \pi}\left[1+\frac{\sigma_{0}}{\left(n_{1}-n_{2}\right)}\left[\frac{\mathrm{d}\left(n_{1}-n_{2}\right)}{\mathrm{d} \sigma}\right]_{\sigma_{0}}\right] .
\end{aligned}
$$

Due to the spectral range $(\Delta u)$ of the source, the integrand (1) differs from zero only for values of $\varepsilon$ belonging to the range $\Delta \varepsilon$ with

$$
\Delta \varepsilon=\pi p \Delta u
$$

Moreover, due to its trend, this integrand (1) have noticeable contribution to the total intensity only in the vicinity of $\pi u p=\ell \pi$, the integer $\ell$ being restricted to values such as $\varepsilon$ belongs to the range $\Delta \varepsilon$ as defined above. Providing that this range is smaller than $\pi$, only the value $k$ is allowed for the integer $\ell$, and the integrand can be restricted to its approximation in the vicinity of the origin for the variable $\varepsilon$, the expansion of the phase retardation $\phi$ being limited to the first order in $\varepsilon$ as well.

Physically, a range $\Delta \varepsilon$ wider than $2 \pi$ would correspond to the order of diffraction $k+1$ overlap- ping the order $k$ : this mathematically as well as physically uncomfortable situation can definitely be prevented by using a Ronchi Ruling $(p=4 b)$ for which every second order vanishes. Thus in this case, the integral to be evaluated is :

$$
\begin{aligned}
I=B_{0} \int \frac{4}{(k \pi)^{2}} \frac{\sin ^{2} N \varepsilon}{\varepsilon^{2}} \times \\
\quad \times[1+\cos (A+2(B+N) \varepsilon)] \mathrm{d} \varepsilon .
\end{aligned}
$$

As assumed by Stockes and Airy, the luminance $B_{0}\left(\sigma_{0}\right)$ of the source has been supposed constant. Providing that its variation with $\sigma_{0}$ is low with respect to that of the cosine in the integrand, that approximation may possibly alter the visibility of the bands but not their position in the spectrum.

The value of this integral can be found in the literature [9] and finally the intensity observed in the screen, while a white source is illuminating the set up, is given by

$$
\left\{\begin{array}{l}
I=I_{0} N \quad B>0 \text { or } B<-2 N \\
I=I_{0}[N+(N-U) \cos A]-2 N<B<0
\end{array}\right.
$$

with

$$
I_{0}=\frac{4 B_{0}}{k^{2} \pi^{2}} ; \quad U=|N-| B||
$$

The $\cos A$ modulation in (8b) corresponds to the bands thus they appear only in case of negative values for $B$ smaller than twice the number $N$ of grating lines covered by the phase plate, their location in the spectrum being determined essentially by the first coefficient $A$ in the expansion of the phase difference.

Our measurements have been focussed onto the position of the bands rather than on their visibility, and subsequently, for the sake of simplicity, the coefficient $B$ is restricted to its first term, the second $\left(\frac{\sigma_{0}}{n} \frac{\mathrm{d} n}{\mathrm{~d} \sigma}\right)$ being a constant usually small $\left(\sim 10^{-2}\right)$ comparatively to the unity. Also it can be attractive to express the intensity (8) in terms of the order of bands defined as follows :

$$
A=2 m \pi \text {. }
$$

Then the coefficient $B$ turns to be

$$
B=\frac{m}{k}
$$

and the intensity on the screen is now given by

$$
\begin{aligned}
& I=I_{0} N \quad \frac{m}{k}>0 ; \frac{m}{k}<-2 N \\
& I=I_{0}\left(N+\left|\frac{m}{k}\right| \cos 2 \pi m\right)-N<\frac{m}{k}<0
\end{aligned}
$$




$$
I=I_{0}\left(N+\left(2 N-\left|\frac{m}{k}\right|\right) \cos 2 \pi m\right) \quad-2 N<\frac{m}{k}<-N
$$

It is worth noting that the negative values for $B$ required for observing the bands can always be achieved by suitably choosing the order of diffraction : for positive orders $m$ of the bands they appear in the negative order $k$ of diffraction and vice versa (experimentally, they will be observed in the side of the higher index plate). Afterwards $m$ and $k$ refer to the absolute values of these orders. The position of the bands in the spectrum is determined by looking for the minimum of the intensity (11). Thus the order $m$ associated with a dark band is solution of the following transcendental equations :

$$
\begin{aligned}
& 2 \pi m=\operatorname{ctg}(2 \pi m) \quad 0<m<k N \\
& 2 \pi(m-2 k N)=\operatorname{ctg}(2 \pi m) \quad k N<m<2 k N .
\end{aligned}
$$

Subsequently a solution of these equations will be refered as capital $M$. As it can be readily seen from a graphical discussion, the actual values for $M$ are very close to half an integer for the medium range but differ from it in the vicinity of 0 and $2 k N$. Missing this deviation while studying the existence of the bands results in some discrepancy between the theoretical forecast and the experimental observation.

That can be an explanation for such a discrepancy reported in a previous paper [8]. Fortunately, as it can be seen in the figure 2 showing the visibility of the bands, that deviation involves the bands on both side of the spectrum where the visibility is low and therefore less suitable for accurate position measurements than the central bands where the visibility is maximum and the associate order $M$ can be classified as half an integer.

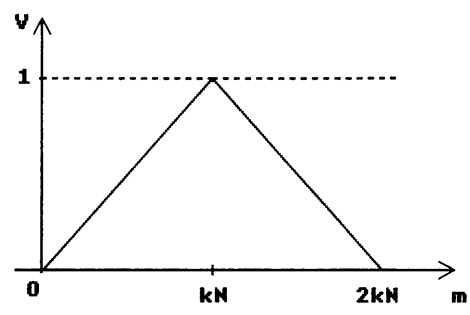

Fig. 2. - Visibility of the bands versus the order of interference $m$.

Thus a dark band is now associated with a definite value for $M$, that means associated with an index value by using the definition (9) (5b). On an other hand, the corresponding wavelength is determined by measuring the position of the band in the spectrum and by using the definition (2b) and (3). Therefore a band is associated to one data point on the dispersion curve. This curve can now be built up the following way : we first record the light intensity in the screen of the spectroscope, the positions of the bands are picked up from this record, converted in wavelength by using relations (2) and (3), the associated index being calculated from relations (9) and (5b). Nevertheless, measuring the dispersion curve by this technique requires the knowledge of at least one order $M$ without ambiguity. This preliminary data can be obtained from either an interferometry experiment under monochromatic light or a known value for the index. This more experimental side is discussed in the next section.

\section{Experimental.}

The experimental set up is a usual grating spectroscope (Fig. 1). A narrow source slit $(25 \mu)$ associated with a large focal length lens $(0.6 \mathrm{~m})$ insure an illuminating beam with a coherence length larger than the beam width limited by a collimating slit (CS). Also due to the large focal length $(1 \mathrm{~m})$ of the output lens $\left(L_{2}\right)$, the relationship between the linear abscissa $x$ in the screen and the wavelength turns to be linear, at least for the first order of diffraction of the grating $(k=1)$

$$
\left(x-x_{0}\right)=\frac{f}{p} \lambda
$$

where $f$ is the focal length of the output lens $L_{2}, x_{0}$ corresponds to the zero diffraction order abscissa. The collimating slit width $L$ is adjusted large enough for the bands to be observable with the maximum of visibility $(L=2 k N p)$. The intensity in the screen has been measured by means of a radiometer with a $10 \mu \mathrm{m}$ wide input slit aperture ; obviously, this width has to be smaller than the band spacing. This radiometer has been mounted on a motorized translation stage, its position being obtained by means of a laser measurement system (HP). First of all the spectroscope has been calibrated by illuminating the set up, free of any phase plate with the red line of a stabilized He-Ne laser. From the recorded intensity curve $I=f(x)$ in these conditions, the abscissa $x_{+}$and $x_{-}$associated with the first orders $(k= \pm 1)$ in the diffraction pattern readily give us the two quantities required in the further processing : the origin $\left(x_{0}\right)$ of the scale and the actual scale factor $f / p$ in the relation (13).

After this calibration has been done, the plate is inserted in such manner than each part covers half the field of the spectroscope now illuminated with 
light source, the visibility being optimized by adjusting the collimating slit width, and then the curve light intensity versus the position is recorded. The figure 3 shows such a curve in case of a plate consisting of a $168 \mu \mathrm{m}$ thick glass (microscope cover slip, Corning $\left({ }^{2}\right)$ ) the resulting dispersion curve is shown in the figure 4 where it can also be seen the manufacturer's data. The reference order $M$ has been evaluated from one of these data. Obviously the index values obtained with our technique are in very good agreement with those of the glass manufacturer ; this is all the more true since the accuracy reported in the curve is rather coarse and pessimistic

$\left.{ }^{2}\right)$ The reference of this glass is 523-602 $\left(n_{\mathrm{d}}=1.52257\right)$. say ten times too large nevertheless it shows that the determination of the dispersion curve for a given material can be done by using the Talbot bands with an accuracy of about $10^{-4}$ which is good enough for most of applications where the index of refraction is involved. It is worth noting that manufacturing the sample requires nothing but a thickness as constant as possible with respect to the wavelength, that means any transparent solid or liquid can be studied by this technique providing the material can easily be sliced. As a result this technique is especially well suitable for studying the liquids. The dispersion of the glass plates limiting the studied liquid does not intervene providing that they cover the whole field of the spectroscope.

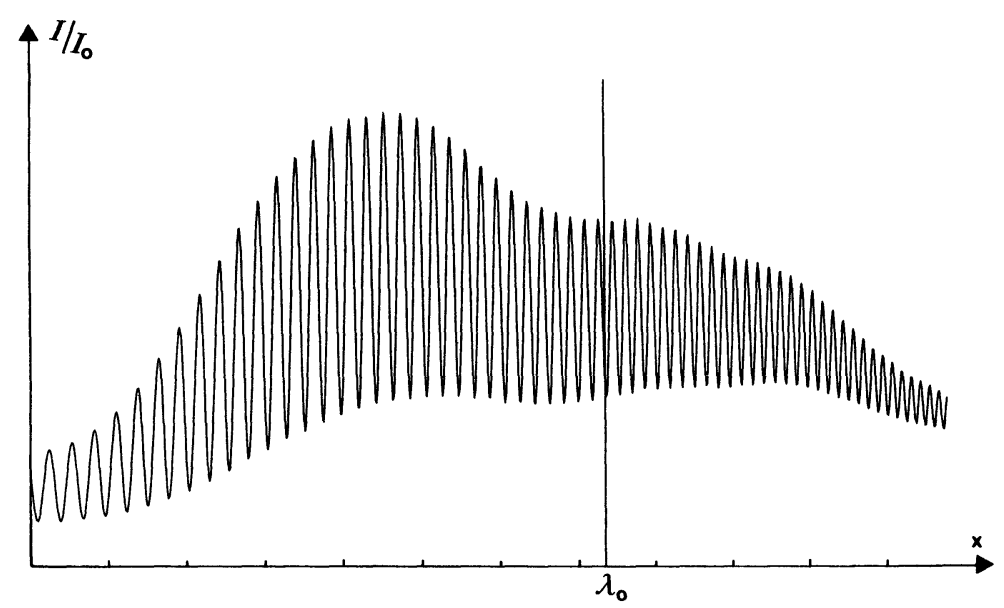

Fig. 3. - Experimental intensity curve in the region where the bands exist. The vertical line corresponds to the calibration red line $0.6328 \mu \mathrm{m}$.

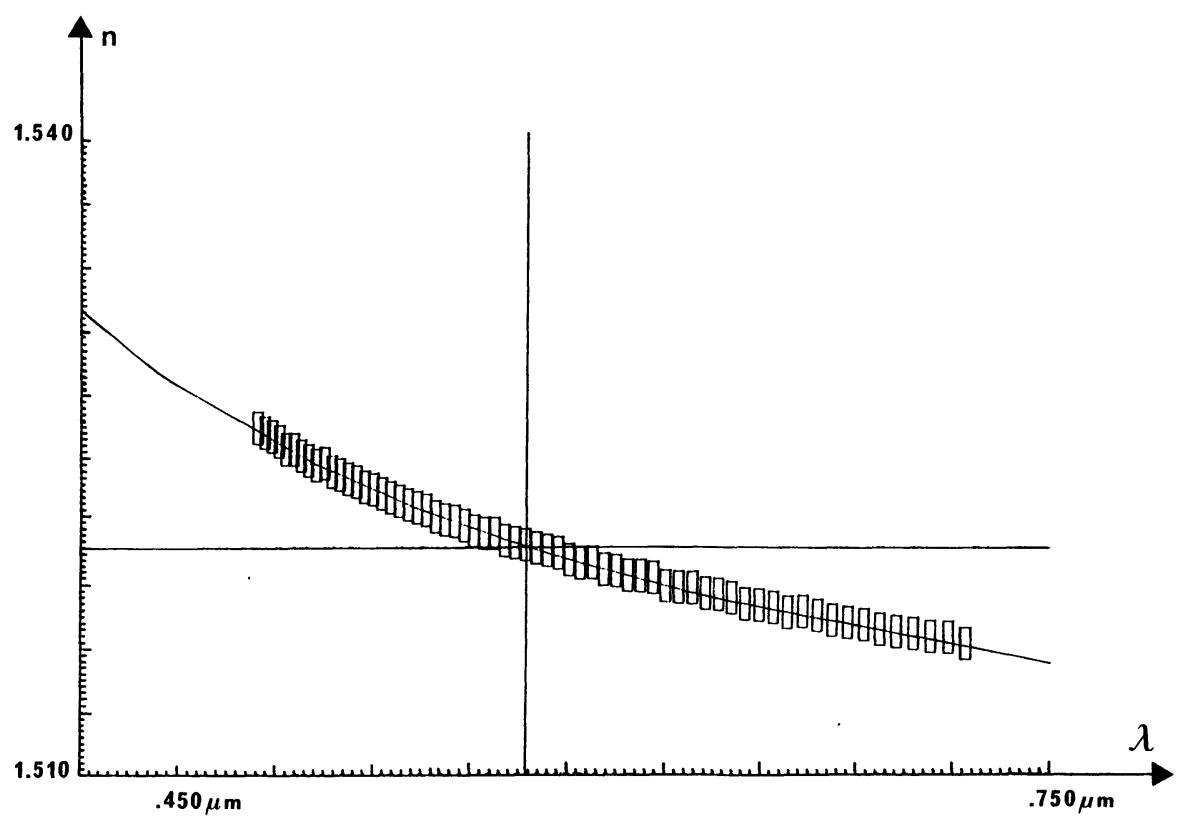

Fig. 4. - Dispersion curve for a microscope cover slip glass (Corning) 168 microns thick. The full lines correspond to the manufacturer's data. 
So far in this paper, the phase plate has been supposed to be made of an isotropic medium, as it will be shown in the next section, the presented technique is still working in case of birefringent material, even both the ordinary and extraordinary index curve can be obtained providing the sample has been properly prepared.

In this first paper, we will only consider the case of a two stepped plate made of a birefringent medium adjacent to an isotropic one. With such a sample, we have to prevent the Talbot bands interference from being mixed with or shaded by the birefringence interferences. This can be experimentally and therefore theoretically achieved by illuminating the spectroscope with a beam either polarized or unpolarized, in the former case one of the principal direction of the crystal and the two polarisers must be parallel. As soon as these conditions are fulfilled, all that have been said in the first section is still valid, the lone question to be solved being what index must appear in the relations (5) between the indices and the order of the bands, in other words what index will be measured.

We first consider the case of a polarized beam. As just said, the analyser has to be parallel to the polarization direction and the in-plane projection of the optical axis must be either perpendicular or parallel to it (Fig. 5). Clearly, in both cases, there exists only one emerging plane wave from the sample associated respectively with the ordinary index and a value defined as below :

$$
n^{2}=\frac{n_{\mathrm{o}}^{2} n_{\mathrm{e}}^{2}}{n_{\mathrm{o}}^{2}+\left(n_{\mathrm{e}}^{2}-n_{\mathrm{o}}^{2}\right) \sin ^{2} \theta}
$$

where $n_{\mathrm{o}}$ and $n_{\mathrm{e}}$ are the principal values for the studied material and $\theta$ is the out of plane tilt angle of the optical axis. Thus, for the extraordinary index to be measured, we have to make up an in-plane optical axis sample with a polarisation parallel to the optical axis. The same sample can be used to obtain the ordinary index dispersion curve, it just takes a

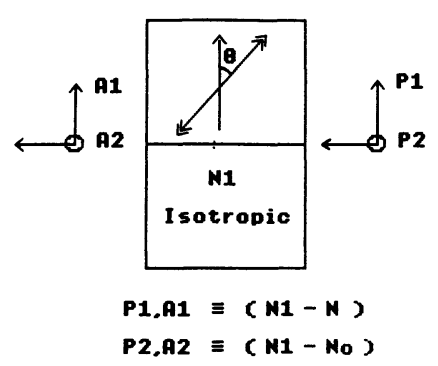

Fig. 5. - Two possible sample geometries for measuring the dispersion curves in case of a birefringent medium illuminated with a polarized beam. $P$ and $A$ refers to the polarizers, the resulting measured index are noted. $\theta$ is the out of plane angle for the optical axis (double arrow). ninety degrees rotation for the polarizers. We now consider the case of an unpolarised beam. Again there exist two situations of special interest according to the optical axis direction parallel or perpendicular to the input plane wave. As this wave is travelling along with the optical axis direction, the medium looks like an isotropic one and the refractive index involved is the ordinary one (Fig. 6). On the contrary, for an input wave travelling perpendicularly to the optical axis direction, there exist two emerging polarized waves (Fig. 7a) associated with the two refractive indices $n_{\mathrm{e}}$ and $n_{\mathrm{o}}$. Due to their respective polarisation, two independent Talbot band patterns appear on the screen of the spectroscope. Although such a situation usually would drastically lower the visibility of the bands and moreover the relationship (5) would not be longer usable, a great advantage can be taken from this situation by suitable choosing

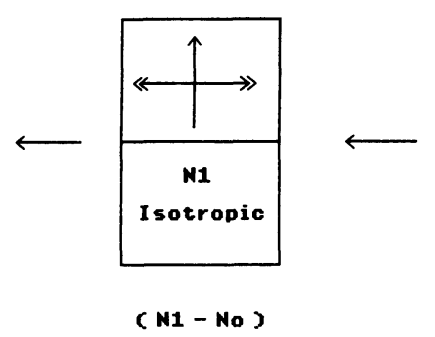

Fig. 6. - Sample geometry for the ordinary index measurement in case of a set up illuminalted with an unpolarized beam. The optical axis is noted with a double arrow.

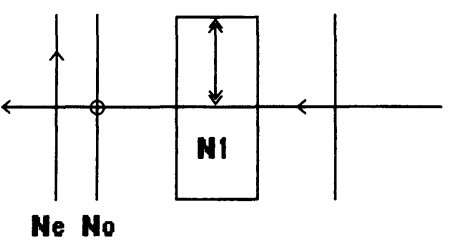

[N1 - Ne] and [N1 - No]

Fig. 7a.

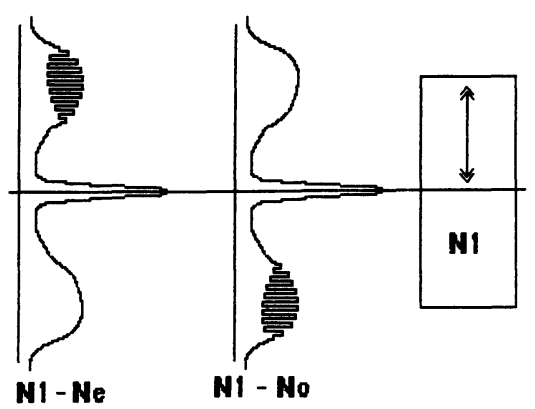

Fig. $7 b$.

Fig. 7. - Simultaneous measurement of ordinary and extraordinary dispersion curves a) emerging waves and their polarization ; b) associated Talbot bands patterns. 
the second isotropic medium that the two stepped plate is made of. Let us remind that the bands only appear in the diffraction pattern in the side where the higher index plate has been set. Let us now suppose that the index $n_{1}$ of the second isotropic plate is such as :

$$
n_{\mathrm{o}}<n_{1}<n_{\mathrm{e}}
$$

and consider the two patterns mentionned above. The ordinary index being smaller than that of the adjacent medium, the associated bands appear in the side of this medium, whereas the bands associated with the extraordinary index must appear in the other side due to a larger index value (Fig. $7 \mathrm{~b}$ ). Making up such a sample allows to separate definitely both patterns and therefore, using the presented technique for each band systems, allows to measure simultaneously the two dispersion curves associated with the extraordinary and ordinary index. The main four situations permitting these curves measurement have been summarized in the figure 8.

They all have been tested successfully with the nematic liquid crystal MBBA (Merck) and compared with previous results obtained by Brunet Germain [10]. The figure 9 shows the ordinary index curve obtained in case of a sample consisting of an air plate $\left(n_{1}=1\right)$ and an homeotropic liquid crystal plate illuminated with an unpolarized beam (case of Fig. 8a), the values from reference (10) are plotted in full line. The figure 10 shows a light intensity record in case of a planar liquid crystal adjacent to a calibrated glass plate (Basf 10, Schott) (case of Fig. $8 \mathrm{~b}$ ). The right and left side bands correspond

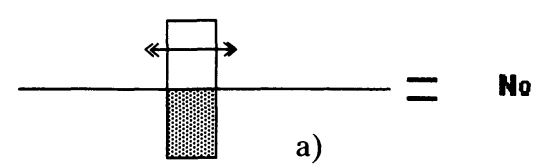

a)
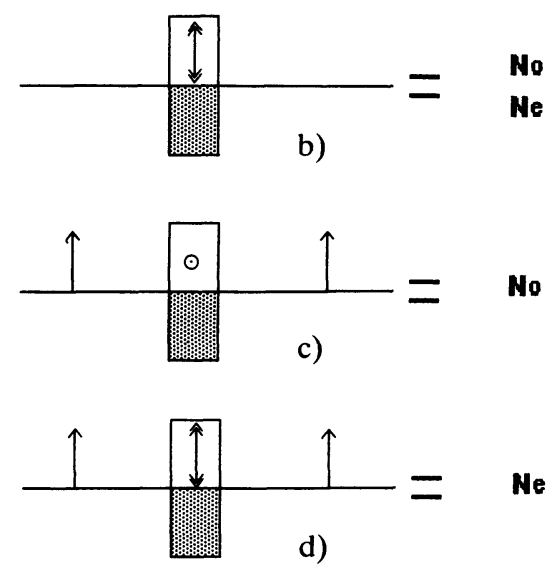

Fig. 8. - Summary of the different sample geometries for the ordinary and extraordinary dispersion curves measurement. The white part of the sample corresponds to the birefringent medium and the grey part to an isotropic one with an intermediate index; explanation in text.

respectively to the extraordinary and ordinary index. The corresponding dispersion curves are shown in the figure 11. Again the values from reference (10) are plotted in full line. The two other ways for measuring these curves have also been tested successfully with the same liquid crystal. Another compound named $\mathrm{E}_{7}(\mathrm{BDH})$ have also been studied, therefore we present the curves for this compound obtained by using płanar samples adjacent to an air

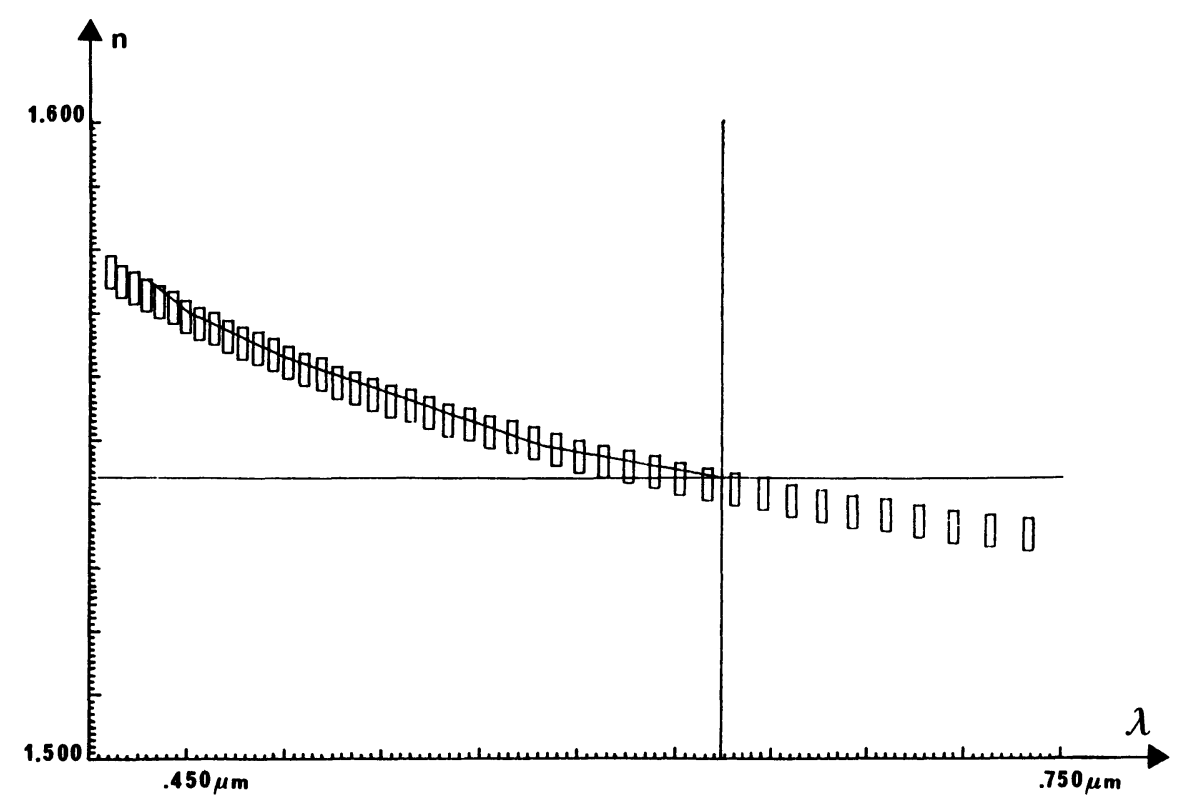

Fig. 9. - Dispersion curve for MBBA (70 $\mu$ thick) obtained as shown in figure 8 a. 


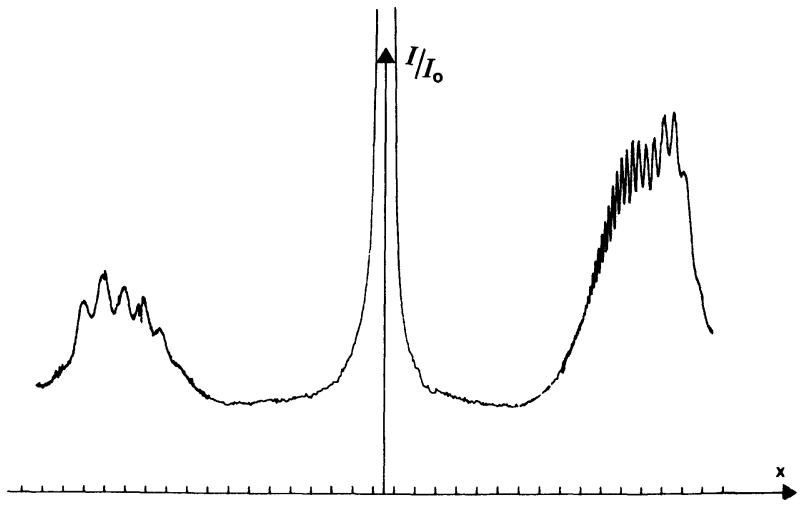

plate $\left(n_{1}=1\right.$; case of Figs. $\left.8 \mathrm{~d}, 8 \mathrm{c}\right)$ and at our knowledge they have not been published so far (Fig. 12).

\section{Conclusion.}

We have presented a new method for studying the wavelength dependence of the refractive index of a

Fig. 10. - Light intensity record obtained with a sample as shown in figure $8 \mathrm{~b}(100 \mu$ thick). The left and right bands correspond respectively to the ordinary and extraordinary waves.
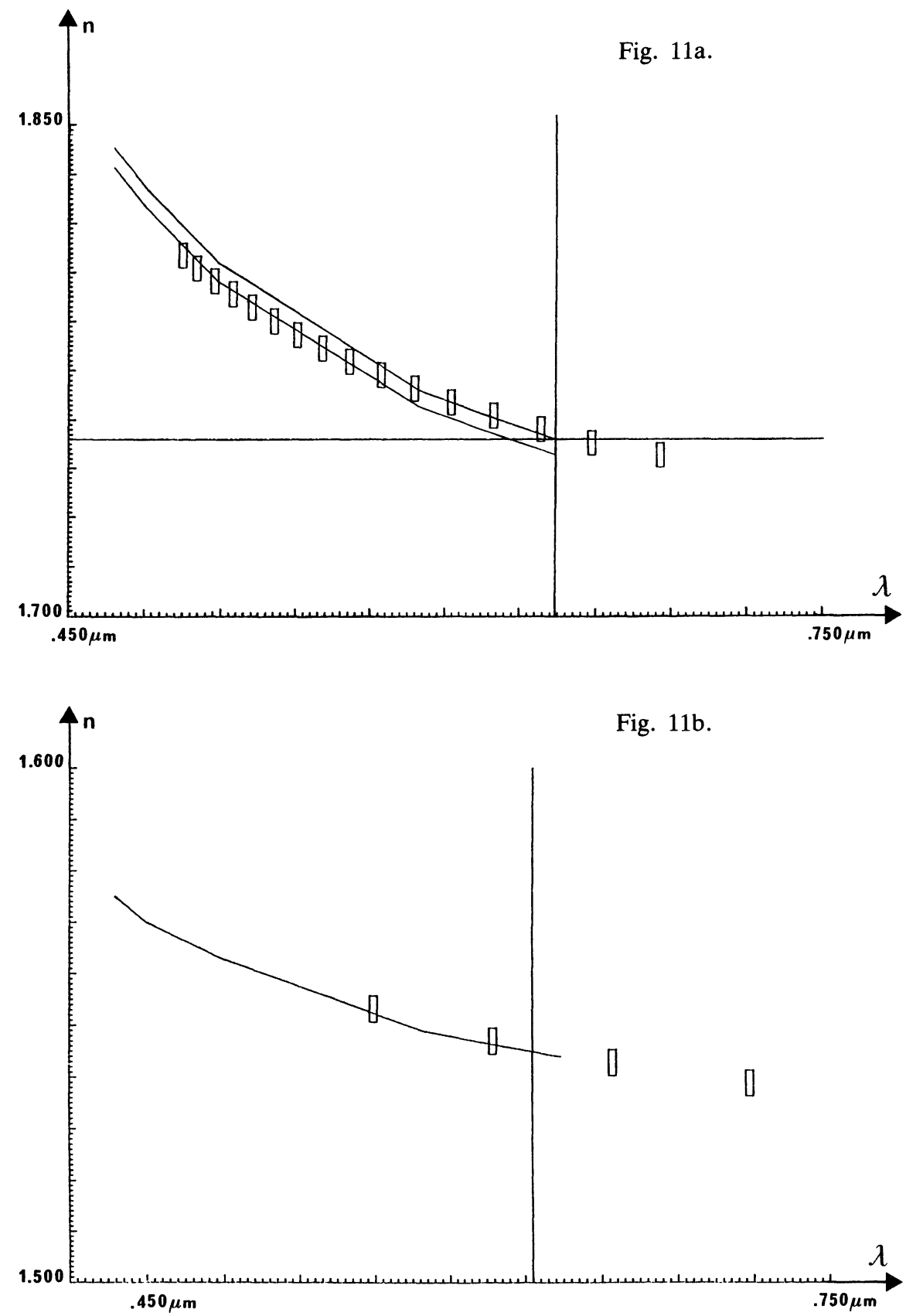

Fig. 11. - Dispersion curves obtained from intensity shown in figure 10. a) Extraordinary index ; b) ordinary index. The full lines corresponds to Brunet Germain data at 2 temperatures [10]. 

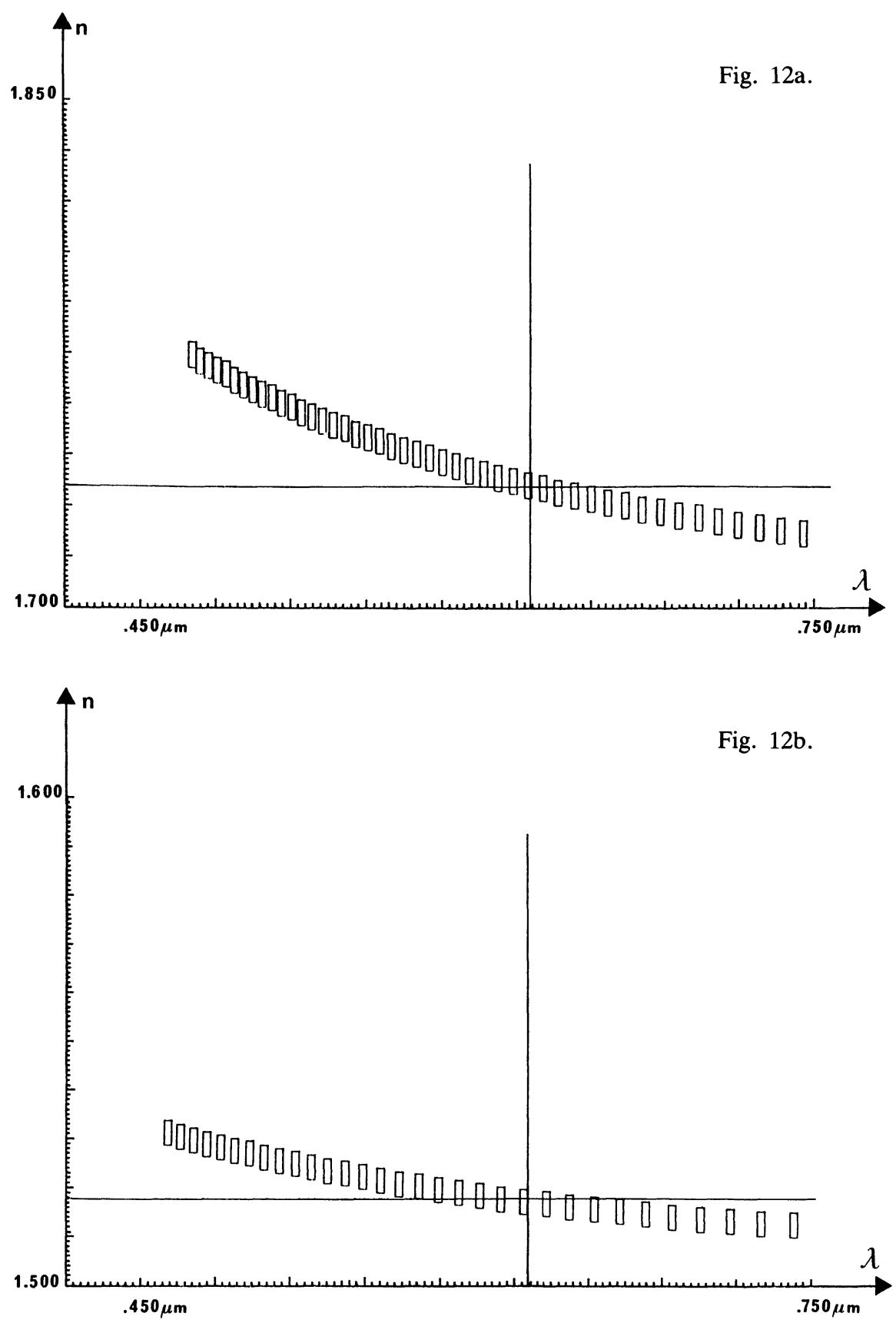

Fig. 12. - Dispersion curve obtained for E7 (78 $\mu)$ with sample geometry 8 b and 8c. a) Extraordinary index ; b) ordinary index.

medium. It consists of an accurate position measurement of dark bands which appear in the diffraction pattern when a phase plate is inserted in the field of a grating spectroscope ; followed by a processing of the data resulting in the dispersion curve determination for the medium the phase plate is made of. This method requires only two expriments, one for the calibration and the other for the measurement itself, moreover about fifty and even more data points can be obtained to build up the dispersion curve, whereas usually it takes as many experiments as data points. Although the accuracy $\left(10^{-4}\right.$,
$10^{-5}$ ) of the results is a bit lower than the usual refractometry measurements $\left(10^{-6}\right)$, it is good enough for most of the application involving the refractive index. From such a curve determination, some usual characteristic quantities, as the Abbe number, can readily be determined. A major interest of the presented technique is its versability since any transparent, isotropic or birefringent, solid or liquid plate can be studied. Especially well suitable for studying the liquids, it has been shown in this paper that the technique is also well adapted to the birefringent medium seeing that both ordinary and 
extraordinary curves can be obtained simultaneously. This technique has been successfully tested for different medium : glass plates and liquid crystal plates, moreover new results are presented for the nematic liquid crystal E7. In case of studying the liquid crystal dispersion curve, it is worth noting the importance of the sample preparation. A perfect molecular orientation both at the surfaces and in the bulk is essential to measure the principal indices, but, on the contrary, an averaged value for the tilt angle of the optical axis (14) can be obtained by fitting curves providing the principal values for the indices are known. Considering the set up, the sample can be easily surrounded by any additional equipment such as an oven and therefore the temperature dependence of the indices can readily be studied within the presented method. Among the different perspectives for using this technique, one can also mention broadening the wavelength range of the source and the optical components and then to determine the dispersion curve in a very large range of wavelength including infrared, far infrared as well as the ultra violet bands; and therefore approach the absorption bands. Finally, in a forthcoming paper, it will be shown that by suitably making up a sample consisting of an isotropic plate adjacent to a liquid crystal plate under the influence of an electric field, it is possible to observe tunable Talbot bands and therefrom to obtain a tunable band pass filter.

\section{Acknowledgment.}

We are indebtful to Mr. G. Valledant from the N.R.C. optical shop for manufacturing the calibrated glass plates.

\section{References}

[1] Calatroni, J., Da Costa, G., Inter. Commun. Opt., Congress 11th Madrid (1978) pp. 643-646.

[2] Talbot, H. F., Philos. Mag. 1 (1837) 364.

[3] Stokes, G. G., Philos. Trans. (1848) p. 227.

[4] AIRY, G. B., Philos. Trans. Roy. Soc. 130 (1840) 225 ; 131 (1841) 1.

[5] Brewster, D., Trans. Brit. Assoc. Adv. Sci. 6 (1837) 12.

[6] Wood, R. W., Physical Optics Mac-Millan (NewYork) pp. 254, 261 (1911).
[7] Longhurst, R. A., Physical Optics Interscience (New-York) 1957, pp. 109-111.

[8] KIng, A. L., Davis, R., Am. J. Phys. 39 (1971) 1195-1198.

[9] Gradshtein, I. S., RyzhiK, I. M., Tables of Integrals series and products (Academic Press) NewYork (1980).

[10] Brunet Germain, M., C.R. Acad. Sci. 271 (1970) 1075. 